\title{
Neoadjuvant imatinib treatment and laparoscopic anus-preserving surgery for a large gastrointestinal stromal tumor of the rectum
}

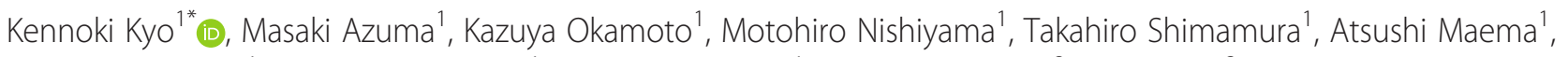 \\ Hitoshi Kanamaru' ${ }^{1}$ Motoaki Shirakawa ${ }^{1}$, Toshio Nakamura', Kazuya Shinmura², Kenji Koda ${ }^{3}$ \\ and Hidetaro Yokoyama ${ }^{1}$
}

\begin{abstract}
Background: Resection of a gastrointestinal stromal tumor (GIST) of the rectum can be difficult because of the particular location in the pelvis, and a large rectal GIST often requires abdominoperineal resection. Recent reports demonstrate that neoadjuvant imatinib treatment improves surgical outcomes in patients with a rectal GIST, and there are only a few reports of the effectiveness of laparoscopic surgery for a rectal GIST.

Case presentation: A 46-year-old man was found to have a rectal GIST that measured $80 \mathrm{~mm}$ and was located on the anterior wall of the lower rectum. After 6 months treatment with imatinib, the tumor decreased in size to $37 \mathrm{~mm}$, and laparoscopic low anterior resection was performed. The patient is currently alive without any evidence of recurrence 37 months after surgery.
\end{abstract}

Conclusions: Neoadjuvant imatinib should be a treatment of choice for a large rectal GIST. When marked tumor shrinkage is achieved, laparoscopic surgery may be the preferred procedure.

Keywords: Gastrointestinal stromal tumor, Neoadjuvant treatment, Rectum, Imatinib, Laparoscopic surgery

\section{Background}

Gastrointestinal stromal tumors (GISTs) are rare tumors with an estimated incidence of $1.5 / 100,000 /$ year but are the most common mesenchymal tumors of the gastrointestinal tract [1]. They are most commonly found in the stomach (60\%) and small intestine (35\%), and $<5 \%$ arise in the rectum [2]. Although complete surgical excision with histologically negative margins is the primary treatment of choice for localized GISTs, resection of rectal GISTs can be difficult in the narrow pelvic space, and a large rectal GIST has a risk of rupture during surgery and often requires abdominoperineal resection. GISTs express KIT proto-oncogenic receptor tyrosine kinase and commonly harbor activating mutations in the KIT

\footnotetext{
*Correspondence: kkyo@vega.ocn.ne.jp

'Department of Surgery, Fujieda Municipal General Hospital, 4-1-11

Surugadai, Fujieda, Shizuoka 426-8677, Japan

Full list of author information is available at the end of the article
}

gene [3]. Imatinib is an inhibitor of tyrosine kinases including KIT, and targets the aberrant signaling pathways that are critical for tumor cell proliferation and survival, thus showing anti-tumor activity [4]. The effectiveness of imatinib in the treatment of GISTs was first described in 2001 [5], and the remarkable response to imatinib in unresectable or metastatic GISTs has led to the neoadjuvant treatment strategy for locally advanced GISTs [6, 7]. Despite the excellent view of laparoscopy, there are only a few that reported cases of its use in surgery for rectal GIST [8, 9]. We report here a patient with a large rectal GIST who safely underwent laparoscopic anus-preserving surgery after neoadjuvant imatinib treatment.

\section{Case presentation}

A 46-year-old man was referred to our hospital because of a 2-month history of anal pain and hematochezia. He also complained of constipation, malaise, and body 
weight loss of $10 \mathrm{~kg}$ in 1.5 years. Computed tomography (CT) and magnetic resonance imaging (MRI) demonstrated a tumor measuring $80 \mathrm{~mm}$ in maximum diameter on the anterior wall of the lower rectum and several enlarged regional nodes (Fig. 1a). No distant metastases were found. Colonoscopic examination revealed a large submucosal tumor on the anterior wall of the rectum just above the dentate line (Fig. 2). Core needle biopsy of the tumor revealed bundles of spindle cells with positive immunohistochemical staining for c-kit antigen (CD117) and CD34, but negative for other differentiation markers such as desmin and S-100 protein (Fig. 3a, b). The immunohistochemical findings led to the diagnosis of a rectal GIST. Neoadjuvant imatinib treatment (400 mg/day) was introduced, and soon after initiation, anal pain was relieved. MRI after 1 month of treatment showed marked shrinkage of the tumor to $52 \mathrm{~mm}$, as well as the lymph nodes. After treatment for 3 and 6 months, the tumor decreased to 44 and $37 \mathrm{~mm}$, respectively (Fig. 1b). CT examination revealed no distant metastases. As a result of the dramatic tumor shrinkage with neoadjuvant treatment and almost maximum tumor response, we decided to operate at this point. The adverse effect of imatinib was fatigue.

After 7 months treatment with imatinib, the patient underwent laparoscopic low anterior resection with total mesorectal excision, coloanal anastomosis, and diverting ileostomy. Imatinib treatment was stopped 3 days before surgery. Gross appearance of the specimen showed a submucosal tumor measuring $43 \times 35 \mathrm{~mm}$, with a 10 $\mathrm{mm}$ distal resection margin (Fig. 4a, b). Histological examinations revealed that the tumor spread within muscularis propria and protruded into submucosal layer in its anal side, with negative resection margins, and demonstrated an excellent response to imatinib with almost complete tumor necrosis (Fig. 4c). Metastasis was not found in the 11 retrieved lymph nodes. Imatinib treatment resumed on postoperative day 7 , but was

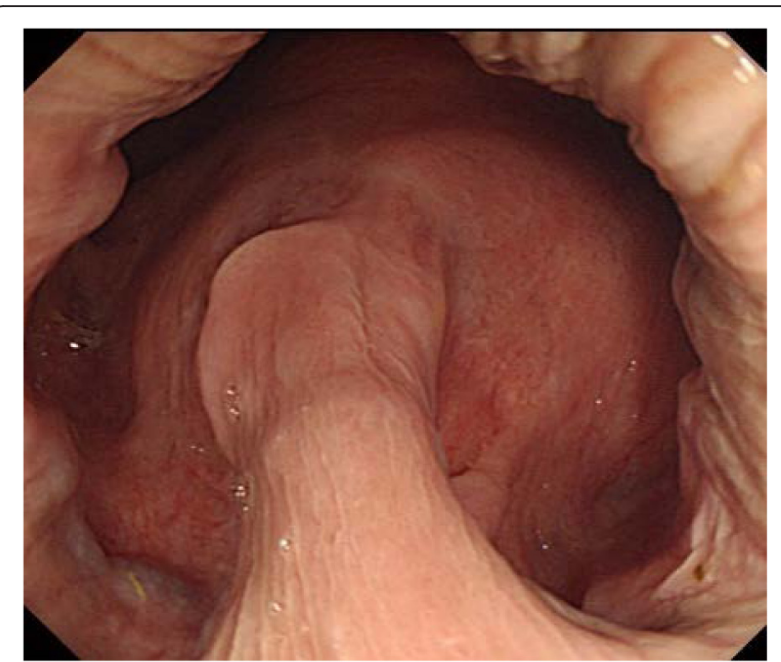

Fig. 2 Colonoscopic image before imatinib treatment. A large submucosal tumor was observed on the anterior wall of the rectum just above the dentate line

interrupted 1 year later because of fatigue. The ileostomy was reversed 3 months after the surgery, and the patient is currently alive without any evidence of recurrence 37 months after surgery.

\section{Discussion}

We safely performed laparoscopic anus-preserving surgery after neoadjuvant imatinib treatment for a large rectal GIST. The primary treatment of choice for patients with localized GISTs is complete surgical excision with negative microscopic margins $[10,11]$. However, in the restricted space of the pelvis, complete resection of a large rectal GIST is difficult and often necessitates abdominoperineal resection, with or without adjacent organ resection. To reduce the size of the tumor, thereby lowering the risk of rupture during surgery, and increasing the likelihood of anus preservation, we introduced neoadjuvant imatinib treatment in our patient. Many studies

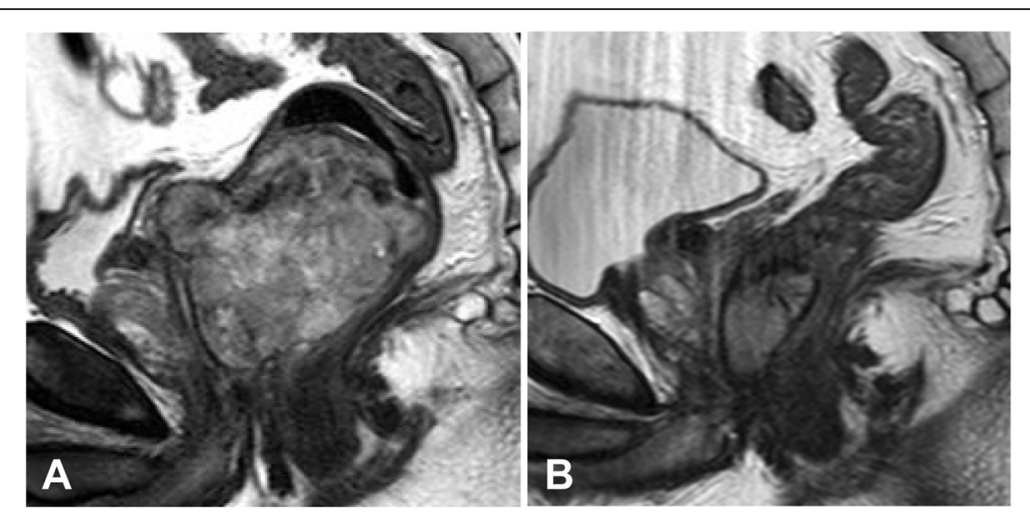

Fig. 1 Sagittal view of pelvic MRI. a Before imatinib treatment, an 80-mm tumor was observed on the anterior wall of the lower rectum. b After 6 months treatment with imatinib, the tumor decreased to $37 \mathrm{~mm}$ 


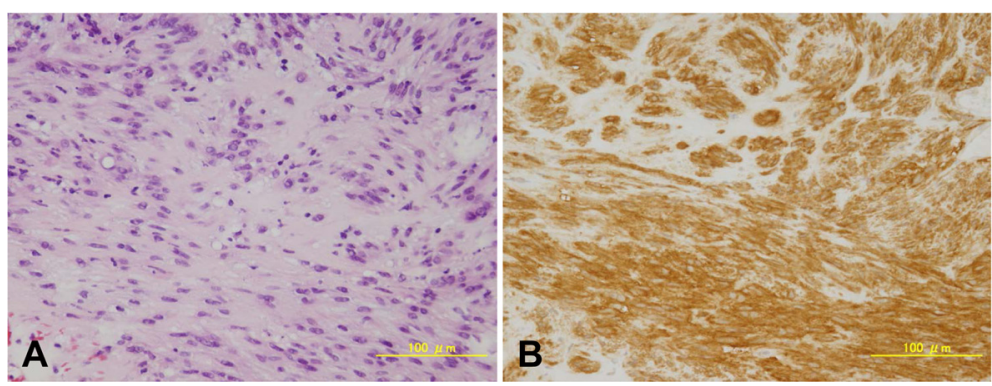

Fig. 3 Core needle biopsy of the tumor. a Hematoxylin and eosin staining. b Immunohistochemical staining for c-kit antigen (CD117)

have shown the effectiveness of imatinib in the treatment of GISTs since the first report in 2001 [5]. Randomized clinical trials have reported response rates of $\sim 70 \%$ in patients with unresectable or metastatic GISTs $[12,13]$. Another study demonstrated that, among 161 patients with locally advanced GISTs who received neoadjuvant imatinib treatment, 129 patients (80.1\%) had a partial response and only two (1.2 \%) showed disease progression during treatment [14]. As for rectal GISTs, several reports demonstrated that neoadjuvant imatinib treatment improved $\mathrm{R} 0$ resection rates and decreased the risk of postoperative morbidity $[6,7]$. There seems to be no worldwide consensus, however, as to the indications for neoadjuvant imatinib treatment, and it is not recommended in the clinical practice guidelines for GIST in Japan [15]. In contrast, in the National Comprehensive Cancer Network guidelines, it is recommended that preoperative imatinib treatment should be considered if abdominoperineal resection is necessary to achieve a negative resection margin, or if the surgeon feels that multivisceral resection may be required [10]. In the European Society for Medical Oncology guidelines, neoadjuvant imatinib is recommended as a standard treatment if R0 surgery can be achieved through function-sparing surgery in the case of cytoreduction, and if the surgeon believes that the procedure is safer after cytoreduction [11].

The clinical response to imatinib is reported to depend on GIST genotype. Mutations in KIT exon 11 are the most common type (65.8-73.1\%), followed by no mutations $(15.2-16.4 \%)$ and mutations in KIT exon 9 (8.28.4\%). Each of the other mutations comprises $\sim 1 \%$ of the total. Mutations in KIT exon 11 correlated with better response rates compared with no mutations and
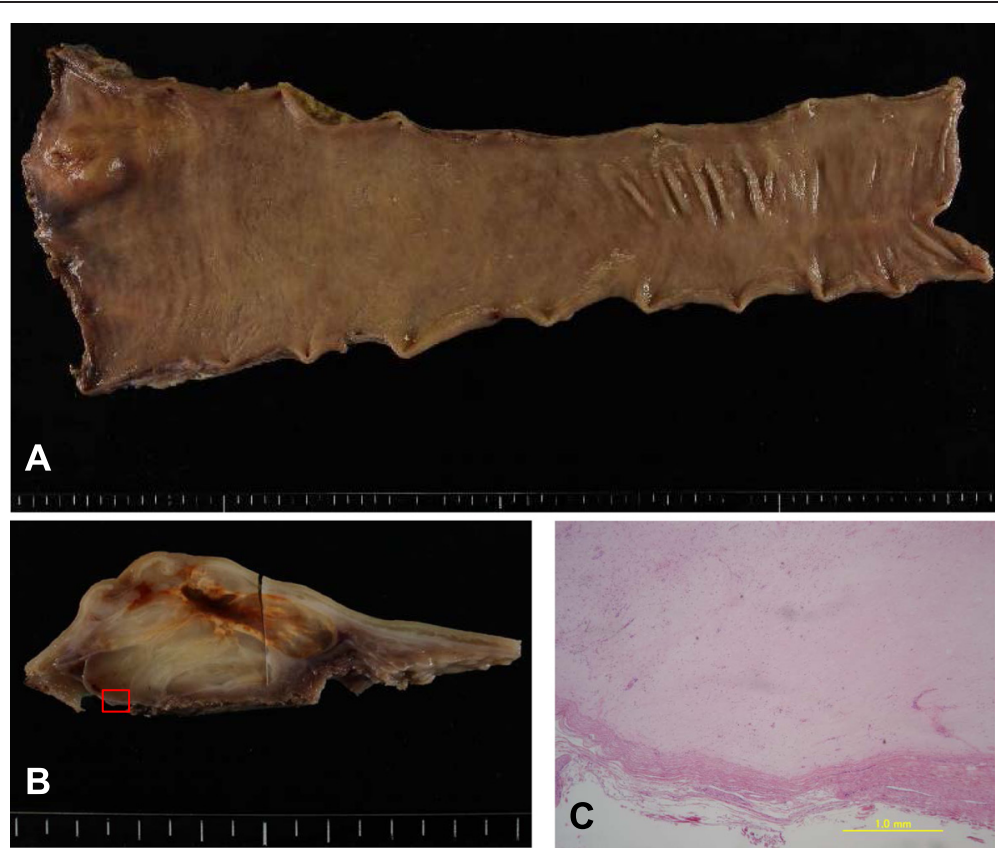

Fig. 4 Pathological examinations. a Gross appearance of the resected specimen. The tumor measured $43 \times 35 \mathrm{~mm}$ in size, and a 10-mm distal resection margin was secured. $\mathbf{b}$ Cross-sectional view of the tumor. Magnified view of the square area is shown in (c). c Histological examination (hematoxylin and eosin staining). All of the tumor cells caused hyaline degeneration 
mutations in KIT exon 9, and the response rates were $69-71.7 \%, 25-44.6 \%$, and 34-44.4 \%, respectively [16, 17]. In patients with KIT exon 9 mutations, high-dose imatinib (800 mg/day) resulted in improved response rates compared with standard-dose therapy (400 $\mathrm{mg} /$ day) [17]. Hence, although mutational analysis is crucial to make a clinical decision about neoadjuvant therapy, the patient required as rapid a start of imatinib treatment as possible, without mutational analysis. This is partly because of the anal pain and the wish to preserve the anus, and partly because of unavailability of the high-dose imatinib regimen in Japan. Instead of the mutational analysis, we carried out close monitoring of the tumor response by MRI, particularly in the early phase of the treatment.

The appropriate timing of surgery is unknown. In randomized clinical studies, the cumulative incidence of response almost reached a plateau after treatment for 68 months, and disease progression occurred in some patients even in this period $[12,16]$. Furthermore, it was demonstrated that the median time to best response was 3.5 months, and little tumor shrinkage was obtained after 9 months of treatment [18]. In the present case, although dramatic tumor shrinkage was obtained after 1 month of treatment, only a little tumor shrinkage was observed after 3 months. This represented almost maximum tumor response; therefore, we decided to operate at this point. Surgery should be done promptly when tumors become safely resectable, before disease progression.

Several approaches exist for resection of rectal GIST, but the principle of surgery is complete resection with an intact pseudo-capsule $[10,11]$. Local resection, such as transanal, trans-sacral, or transvaginal, may be suitable for small tumors, but for larger ones, radical resection, such as low anterior resection is preferable. Several studies have demonstrated the association between local resection and local recurrence $[7,19]$. One study showed that, among the 36 patients who underwent surgery for rectal GIST, all five who developed local recurrence had undergone local resection with positive margins, and without perioperative imatinib treatment [7]. Radical resection was more likely to have resulted in negative resection margins (R0) than local resection (13 of 15 vs. 11 of $21, P=0.03$ ), although the radical resection group had larger tumors. Another study also demonstrated that the local recurrence rate after local resection was $77 \%$ compared with $31 \%$ after radical resection, although the tumors were smaller in local resection group [19].

The remarkable response to imatinib enabled us to perform laparoscopic surgery safely. Several studies have demonstrated that laparoscopic resection for a small- or medium-sized gastric GIST is safe and effective when performed by an expert surgeon [20]. However, there are only a few reports of the effectiveness of laparoscopic surgery for a rectal GIST; partly because of the rarity of the disease $[8,9]$. Although open radical resection for rectal GIST resulted in lower rates of involved resection margins and local recurrence than local resection, they still showed high values $[7,19]$. This is at least partly because of the poor visualization in the deep narrow pelvis. Laparoscopy provides an excellent magnified view in the deep confined space of the pelvis; therefore, laparoscopic surgery should improve surgical outcomes and may be the preferred procedure in patients with small- or medium-sized rectal GISTs. Although we performed total mesorectal excision to dissect the enlarged nodes detected before the neoadjuvant treatment, the incidence of node involvement has been reported as 5.0-8.8 \% [21, 22], and lymphadenectomy is usually not required $[10,11]$.

\section{Conclusions}

Neoadjuvant imatinib should be a treatment of choice for large rectal GIST to increase the chance of anal preservation, and achieve surgical safety. When dramatic tumor shrinkage is obtained, laparoscopic surgery may be the preferred procedure.

\section{Consent}

Written informed consent was obtained from the patient for publication of this Case report and accompanying images. A copy of the written consent is available for review by the Editor-in-Chief of this journal.

\section{Abbreviations \\ CT: computed tomography; GIST: gastrointestinal stromal tumor; MRI: magnetic resonance imaging.}

\section{Competing interests}

The authors declare that they have no competing interests.

\section{Authors' contributions}

KKyo wrote the manuscript. KKyo and MA performed the operation. KS and KKoda carried out the histological studies. MN, KO, TS, AM, HK, MS, TN, and $\mathrm{HY}$ reviewed the manuscript. All authors read and approved the final manuscript

\section{Author details}

'Department of Surgery, Fujieda Municipal General Hospital, 4-1-11 Surugadai, Fujieda, Shizuoka 426-8677, Japan. ${ }^{2}$ Department of Pathology, Hamamatsu University School of Medicine, 1-20-1, Handayama, Higashi-ku, Hamamatsu, Shizuoka 431-3192, Japan. ${ }^{3}$ Department of Pathology, Fujieda Municipal General Hospital, 4-1-11 Surugadai, Fujieda, Shizuoka 426-8677, Japan.

Received: 8 July 2015 Accepted: 4 March 2016

Published online: 08 March 2016

References

1. Gatta G, van der Zwan JM, Casali PG, Siesling S, Dei Tos AP, Kunkler I, et al. Rare cancers are not so rare: the rare cancer burden in Europe. Eur J Cancer. 2011;47:2493-511.

2. Miettinen $M$, Lasota J. Gastrointestinal stromal tumors: review on morphology, molecular pathology, prognosis, and differential diagnosis. Arch Pathol Lab Med. 2006;130:1466-78.

3. Hirota S, Isozaki K, Moriyama Y, Hashimoto K, Nishida T, Ishiguro S, et al. Gain-of-function mutations of c-kit in human gastrointestinal stromal tumors. Science. 1998;279:577-80. 
4. Buchdunger E, Cioffi CL, Law N, Stover D, Ohno-Jones S, Druker BJ, et al. Abl protein-tyrosine kinase inhibitor STI571 inhibits in vitro signal transduction mediated by c-kit and platelet-derived growth factor receptors. J Pharmacol Exp Ther. 2000:295:139-45.

5. Joensuu H, Roberts PJ, Sarlomo-Rikala M, Andersson LC, Tervahartiala P, Tuveson D, et al. Effect of the tyrosine kinase inhibitor ST1571 in a patient with a metastatic gastrointestinal stromal tumor. N Engl J Med. 2001;344:1052-6.

6. Machlenkin S, Pinsk I, Tulchinsky H, Ziv Y, Sayfan J, Duek D, et al. The effect of neoadjuvant imatinib therapy on outcome and survival after rectal gastrointestinal stromal tumour. Colorectal Dis. 2011;13:1110-5.

7. Jakob J, Mussi C, Ronellenfitsch U, Wardelmann E, Negri T, Gronchi A, et al. Gastrointestinal stromal tumor of the rectum: results of surgical and multimodality therapy in the era of imatinib. Ann Surg Oncol. 2013;20:586-92.

8. Nozawa H, Kanazawa T, Tanaka T, Takahashi M, Ishihara S, Sunami E, et al. Laparoscopic resection of a gastrointestinal stromal tumor of the lower rectum in a patient with coronary artery disease following long-term neoadjuvant imatinib treatment and anticoagulation therapy. World J Surg Oncol. 2014;12:211

9. Akiyoshi T, Ueno M, Fukunaga Y, Nagayama S, Fujimoto Y, Konishi T, et al, Laparoscopic local excision and rectoanal anastomosis for rectal gastrointestinal stromal tumor: modified laparoscopic intersphincteric resection technique. Dis Colon Rectum. 2014;57:900-4.

10. National Comprehensive Cancer Network. NCCN Clinical Practice Guidelines in Oncology. Soft Tissue Sarcoma. Version 1. 2015 http://www.nccn.org/ professionals/physician_gls/pdf/sarcoma.pdf.

11. The ESMO/European Sarcoma Networking Group. Gastrointestinal stromal tumours: ESMO Clinical Practice Guidelines for diagnosis, treatment and follow-up. Ann Oncol. 2014;25 Suppl 3:iii21-6.

12. Nishida T, Shirao K, Sawaki A, Koseki M, Okamura T, Ohtsu A, et al. Efficacy and safety profile of imatinib mesylate (ST1571) in Japanese patients with advanced gastrointestinal stromal tumors: a phase II study (ST1571B1202). Int J Clin Oncol. 2008;13:244-51.

13. Blanke CD, Demetri GD, von Mehren M, Heinrich MC, Eisenberg B, Fletcher JA, et al. Long-term results from a randomized phase II trial of standard- versus higher-dose imatinib mesylate for patients with unresectable or metastatic gastrointestinal stromal tumors expressing KIT. J Clin Oncol. 2008;26:620-5.

14. Rutkowski P, Gronchi A, Hohenberger P, Bonvalot S, Schöffski P, Bauer S, et al. Neoadjuvant imatinib in locally advanced gastrointestinal stromal tumors (GIST): the EORTC STBSG experience. Ann Surg Oncol. 2013;20:2937-43.

15. Nishida T, Hirota S, Yanagisawa A, Sugino Y, Minami M, Yamamura Y, et al. Clinical practice guidelines for gastrointestinal stromal tumor (GIST) in Japan: English version. Int J Clin Oncol. 2008;13:416-30.

16. Debiec-Rychter M, Sciot R, Le Cesne A, Schlemmer M, Hohenberger P, van Oosterom AT, et al. KTT mutations and dose selection for imatinib in patients with advanced gastrointestinal stromal tumours. Eur J Cancer. 2006;42:1093-103.

17. Heinrich MC, Owzar K, Corless CL, Hollis D, Borden EC, Fletcher CD, et al. Correlation of kinase genotype and clinical outcome in the North American Intergroup Phase III Trial of imatinib mesylate for treatment of advanced gastrointestinal stromal tumor: CALGB 150105 Study by Cancer and Leukemia Group B and Southwest Oncology Group. J Clin Oncol. 2008:26:5360-7.

18. Verweij J, Casali PG, Zalcberg J, LeCesne A, Reichardt P, Blay JY, et al. Progression-free survival in gastrointestinal stromal tumours with high-dose imatinib: randomised trial. Lancet. 2004;364:1127-34.

19. Changchien CR, Wu MC, Tasi WS, Tang R, Chiang JM, Chen JS, et al. Evaluation of prognosis for malignant rectal gastrointestinal stromal tumor by clinical parameters and immunohistochemical staining. Dis Colon Rectum. 2004:47:1922-9.

20. Novitsky YW, Kercher KW, Sing RF, Heniford BT. Long-term outcomes of laparoscopic resection of gastric gastrointestinal stromal tumors. Ann Surg. 2006;243:738-45.

21. Woodall 3rd CE, Brock GN, Fan J, Byam JA, Scoggins CR, McMasters KM, et al. An evaluation of 2537 gastrointestinal stromal tumors for a proposed clinical staging system. Arch Surg. 2009;144:670-8.

22. Tokunaga M, Ohyama S, Hiki N, Fukunaga T, Yamamoto N, Yamaguchi T. Incidence and prognostic value of lymph node metastasis on c-Kit-positive gastrointestinal stromal tumors of the stomach. Hepatogastroenterology. 2011;58:1224-8.

\section{Submit your next manuscript to BioMed Central and we will help you at every step:}

- We accept pre-submission inquiries

- Our selector tool helps you to find the most relevant journal

- We provide round the clock customer support

- Convenient online submission

- Thorough peer review

- Inclusion in PubMed and all major indexing services

- Maximum visibility for your research

Submit your manuscript at www.biomedcentral.com/submit

) Biomed Central 